\title{
TTR
}

Traduction, terminologie, re?daction

\section{Henri G. Schogt. Linguistics, Literary Analysis, and Literary Translation. University of Toronto Press, 1988, 177 pages.}

\section{Antoine Di-Lillo}

Volume 3, numéro 2, 2e semestre 1990

La traduction des textes sacrés : le domaine biblique

URI : https://id.erudit.org/iderudit/037076ar

DOI : https://doi.org/10.7202/037076ar

Aller au sommaire du numéro

Éditeur(s)

Association canadienne de traductologie

ISSN

0835-8443 (imprimé)

1708-2188 (numérique)

Découvrir la revue

Citer ce compte rendu

Di-Lillo, A. (1990). Compte rendu de [Henri G. Schogt. Linguistics, Literary Analysis, and Literary Translation. University of Toronto Press, 1988, 177 pages.] TTR, 3(2), 145-148. https://doi.org/10.7202/037076ar

Tous droits réservés ( C TTR: traduction, terminologie, rédaction — Les auteurs, Ce document est protégé par la loi sur le droit d'auteur. L’utilisation des 1990 d'utilisation que vous pouvez consulter en ligne.

https://apropos.erudit.org/fr/usagers/politique-dutilisation/ 
la non-satisfaction d'un acte illocutoire puisse limiter ce qui existe est aller trop loin.

[...] le fait que des actes illocutoires de certaines formes aient des conditions de succès ou de satisfaction plus fortes que d'autres montre qu'il existe un ordre a priori de la pensée et du monde. (p. 10)

Nous admettons la relation établie entre les conditions de succès et de satisfaction des actes illocutoires avec les formes $a$ priori de la pensée, mais nous ne sommes pas en mesure d'accepter que de telles conditions révèlent nécessairement un ordre $a$ priori du monde. Il est possible que le monde n'ait aucun principe d'ordre en lui-même et que ce ne soit que nous, en tant qu'être rationnels, qui puissions lui imposer des formes de rationalité.

Ces points de désaccord - parmi d'autres - relatifs aux présupposés théoriques de Daniel Vanderveken et aux nôtres exigeraient de plus amples développements. Il reste que c'est là un travail excellent qui suscite l'admiration pour la rigueur, l'originalité et son apport immense aux recherches dans le domaine de la philosophie, de la logique et de toutes les sciences du langage et de la cognition. Il s'agit d'un ouvrage dont la lecture est obligatoire pour tous ceux qui travaillent dans ces domaines.

Vera Vidal

Université Fédérale de

Rio de Janeiro

Henri G. SCHOGT. Linguistics, Literary Analysis, and Literary Translation. University of Toronto Press, 1988, 177 pages.

L'auteur se penche sur les relations qui existent entre la linguistique, l'analyse littéraire et la traduction littéraire. Plus spécifiquement, il tente de mettre en évidence l'apport de chacune de ces disciplines aux autres. Il indique clairement que le but de l'ouvrage n'est pas de défendre une quelconque théorie, ni de donner une description exhaustive d'un modèle littéraire ou linguistique pour l'analyse de textes. Il vise plutôt à découvrir le rôle joué par 
certaines caractéristiques de différents modèles dans la traduction d'œuvres littéraires.

Avant d'aborder de front ces questions, l'auteur procède à la caractérisation des différents domaines d'étude en jeu: domaines de la linguistique, de la théorie, de la traduction, de l'analyse littéraire et de l'interprétation de textes. Il établit les rapports qu'entretiennent, d'une part, les notions de systèmes, de norme et d'usage et, d'autre part, celles de littérature, de norme, d'usage et d'évaluation. Font également l'objet d'un examen le signe linguistique et les champs notionnels, l'arbitraire du signe, la convention et la motivation lexicale, les universaux. Autant de notions qui ne sont pas toujours très faciles à cerner.

A juste titre, Schogt dénonce, exemples à l'appui, la confusion qui règne dans l'emploi du vocabulaire métalinguistique, qu'il s'agisse d'emprunts interdisciplinaires ou tout simplement dans un même domaine (par exemple, des termes utilisés en linguistique ont parfois un sens différent selon les écoles de linguistique qui les emploient).

Schogt étudie la fonction qu'ont les noms de personnages dans les æuvres littéraires. Il y voit plus que de simples étiquettes. Il est d'avis que des liens inconscients existent entre ces noms et les noms des personnes qui ont joué un rôle dans la vie des auteurs. D'autre part, les stéréotypes (qui existent aussi dans les noms) déclenchent certaines attentes chez le lecteur; les auteurs ont recours à ces stéréotypes pour créer une impression de réalité. Comment peut-on parvenir à garder associations et connotations dans la traduction?

Sur les 177 pages que compte le livre de Schogt, environ 80 pages sont consacrées à l'examen des notions dont nous venons de parler et au débroussaillage de questions qui leur sont liées. C'est à la lumière des résultats de cette étude que Schogt pourra tirer des conclusions lui permettant d'établir les rapports qui existent entre la linguistique, l'analyse littéraire et la traduction littéraire.

Pour répondre à la question de l'apport de la linguistique à l'analyse littéraire, l'auteur affirme que la première discipline peut offrir à la seconde un modèle pour le traitement des données langagières, une terminologie bien définie (ce qui pourrait être contredit par la «confusion qui règne dans le vocabulaire métalin- 
guistique») et des affirmations vérifiables. Ce sont surtout, les études sémantiques et, plus récemment, sociolinguistiques qui pourraient permettre aux analyses littéraires de parvenir à une meilleure compréhension des raisons pour lesquelles un auteur a pu choisir entre certaines formes.

Par contre, la linguistique ne peut apporter de réponse aux problèmes que posent les connotations et les associations touchant un texte. De plus cette discipline ne peut donner de réponse nette à un analyste littéraire qui se demande si, dans certains cas particuliers, il a affaire, ou non, à l'expressivité ou à l'hypersémantisation (cas où le code conventionnel est modifié et où le lecteur doit deviner le sens des formes pour comprendre le texte).

De son côté, l'analyse littéraire se révèle de peu d'utilité pour le linguiste. Toutefois, le linguiste qui veut étudier une langue ancienne peut profiter de l'existence de textes littéraires d'époque. Mais c'est en «se frottant» à l'analyse littéraire que la linguistique va pouvoir mieux définir son champ de compétence. Ainsi, les notions d'intertextualité, d'association et de connotation ne pouvant s'exprimer par des formules linguistiques, la linguistique doit surtout ramener son objet d'étude à la dénotation.

Quant à l'apport de la linguistique à la traduction, Schogt conclut que la linguistique fournit au traducteur une description typologique qui lui permet de comparer les façons dont les différentes langues expriment certaines actions et relations fondamentales. De plus, l'analyse contrastive aboutit à une comparaison entre les champs sémantiques contribuant souvent à la découverte de meilleurs équivalents dans la langue-cible. Schogt présente des exemples où la linguistique peut révéler le problème qui existe lorsqu'un terme dans une langue correspond à deux termes dans l'autre langue. Ce problème est plus grave lorsque c'est la languesource qui n'a qu'un terme.

Mais son analyse tend à montrer que la linguistique, à part le fait de mettre en évidence les problèmes, n'apporte aucune solution générale valable à la traduction. Ainsi, lorsqu'il y a un lien expressif ou onomatopéique dans la langue-source, par exemple, la linguistique peut le déceler et peut déterminer si le signifiant est motivé ou non, mais ne va pas plus loin. L'auteur nous montre que les formes dialectales ou étrangères peuvent aussi être décrites par la linguistique sans pour autant être traitées par cette même discipline. 
Si l'on se tourne maintenant vers l'apport de la traduction à la linguistique, l'étude de Schogt indique qu'elle n'est pas très importante. En effet, lorsque les traductions littéraires sont utilisées par des linguistes, ce qui est rarement le cas, elles leur révèlent qu'il y a différentes façons de dire la même chose. Toutefois, il est montré que la comparaison entre un texte-source où l'expressivité sonore est très importante et un texte-cible où seule la dénotation est transmise remet en question l'arbitraire de la relation signifiantsignifié.

Pour ce qui est de l'apport de l'analyse littéraire à la traduction, Schogt s'attache à montrer que généralement l'analyse littéraire offre aux traducteurs une interprétation du texte-source en se fondant sur les différents aspects du texte analysé. Le traducteur devrait en principe tenir compte dans le texte-cible des différents éléments mis en lumière. Toutefois, déplore l'auteur, il s'avère que l'analyse littéraire peut, dans certains cas, compliquer la tâche du traducteur, particulièrement de celui qui désire suivre le «servile path» (terme que Schogt emprunte à Nabokov), attitude qui consiste pour le traducteur à s'abstenir de toute créativité et à ne pas se substituer à l'auteur de l'œuvre d'origine.

Par contre, l'apport de l'activité traduisante à l'analyse littéraire, aussi bien que la comparaison d'un texte original avec ses traductions, peuvent faire ressortir, veut montrer Schogt, des éléments important du texte-source qui auraient pu autrement passer inaperçus.

Voilà, d'une façon très schématique, les principaux aspects abordés par l'auteur et les principales conclusions auxquelles il aboutit. Le livre est divisé en 18 chapitres courts (de 5 à 8 pages en général). Les notes sont abondantes, elles occupent environ 30 pages. Il est intéressant de souligner que l'auteur étaye ses affirmations par des exemples empruntés à la littérature russe, française et canadienne. Plusieurs exemples d'œuvres québécoises traduites en anglais sont cités (la Sagouine d'Antonine Maillet, la Guerre Yes Sir! de Rock Carrier, etc.). Ceux que le sujet intéresse gagneraient à parcourir cet ouvrage écrit sans prétention et généralement facile à lire. 